\title{
基于图像语义的弱监督显著性物体检测
}

\author{
赵世敏 ${ }^{1)}$ ，王鹏杰 $\left.{ }^{1,2}\right)^{*}$ ，曹乾 ${ }^{1)}$ ，宋海玉 ${ }^{1)}$ ，李威 ${ }^{1)}$ \\ ${ }^{1)}$ (大连民族大学计算机科学与工程学院 大连 116000) \\ ${ }^{2)}$ (大连理工大学信息与通信工程学院 大连 116024) \\ (pengjiewang@gmail.com)
}

摘 要: 为了减少显著性物体检测对像素级标签的依赖, 提出了一种基于图像语义的弱监督显著性物体检测方法. 利用鱼网络和注意力机制的组合模型，在图像语义热力映射图的基础上，对弱标签采用余弦相似度进行训练更新， 同时在网络训练初期采用训练诱导策略，利用简单数据集对整个网络进行诱导训练，使其具有一定的能力. 然后，经 过不断地增加数据集的复杂性, 使得网络提取特征的能力越来越强. 在 4 个显著性检测数据集上进行实验, 并与传统 监督方法进行对比分析, 实验结果表明, 该方法的 $F$-MAX 值在各个数据集上平均提高 $0.03 \sim 0.08$, MAE 减少 $0.02 \sim 0.05$, 在较弱的监督标签下能更精确地提取图像中的显著性特征.

关键词：组合模型；语义映射图；余弦相似度；诱导训练

中图法分类号: TP391.41 DOI: 10.3724/SP.J.1089.2021.18318

\section{Weakly Supervised Salient Object Detection Based on Image Semantics}

\author{
Zhao Shimin $^{1)}$, Wang Pengjie ${ }^{1,2)^{*}}$, Cao Qian ${ }^{1)}$, Song Haiyu ${ }^{1)}$, and Li Wei ${ }^{1)}$ \\ 1) (School of Computer Science and Technology, Dalian Minzu University, Dalian 116000) \\ ${ }^{2)}$ (School of Information and Communication Engineering, Dalian University of Technology, Dalian 116024)
}

\begin{abstract}
To reduce the dependence of salient object detection on pixel-level labels, we propose a weakly supervised salient object detection method based on image semantics. Using the combined model of the fish network and the attention mechanism, on the basis of the image semantic heat map, the weak labels were trained and updated by cosine similarity. At the same time, we used a training induction strategy. In the initial stage of network training, a simple dataset was used to induce the entire network to make it have certain capabilities. Then, after continuously increasing the complexity of the dataset, the network's ability to extract features became stronger and stronger. Experiments were conducted on four saliency detection data sets, and compared with traditional supervision methods. The experimental results show that the $F$-MAX value of this method is increased by $0.03-0.08$ on each data set on average, and the MAE is reduced by 0.02-0.05. Under the weak supervision label, this method can more accurately extract the salient features.
\end{abstract}

Key words: combination model; semantic mapping; cosine similarity; induction training

收稿日期：2020-04-15; 修回日期：2020-09-10. 基金项目：国家自然科学基金(61300089); 辽宁省高等学校创新人才支持计划 (LR2016071); 大连民族大学服务国家战略专项(2020fwgj001). 赵世敏(1994一), 男, 硕士研究生, 主要研究方向为计算机视觉; 王鹏杰 (1978一), 男, 博士, 教授, 硕士生导师, CCF 会员, 论文通讯作者, 主要研究方向为视觉显著性、计算机图形学; 曹乾(1996一), 男, 硕士研究生, 主要研究方向为计算机视觉; 宋海玉(1971-), 男, 博士, 副教授, 硕士生导师, 主要研究方向为计算机视觉; 李威 (1978-), 女, 硕士, 讲师, 主要研究方向为计算机视觉. 
计算机视觉各项研究在深度神经网络的快速 发展下取得了极大的进步。显著性物体检测是计 算机视觉中的一个基本问题，其旨在对图像中最 明显的区域进行定位和分割. 近年来，由于它在计 算机视觉应用中具有较大的实用性而受到了广泛 的关注 ${ }^{[1-3]}$. 最初利用像素级别的标注标签对网络 进行训练能够得到细粒度的物体轮廓, 且能够很 好地捕获图像中语义比较突出的区域，比无监督 方法 ${ }^{[4-5]}$ 所表达的信息更加丰富和完整. 然而高效 的训练标签需要花费极大的精力去标注，为了满 足网络的大规模训练，人们开始采用图像级别的 标签进行微调像素级别的模型训练任务，而图像 级别的标签是比较容易收集的. 图像特征中语义 是具有高级别关系的特征，如何利用图像级标签 去挖掘图像所包含的高级语义信息是近年来弱监 督学习中研究的重点. Long 等 ${ }^{[6]}$ 认为仅仅通过图像 类别训练的深度神经网络可以记住图像中物体的 位置信息，这种特征对于弱监督的网络训练中具 有很好的特征引导作用，有利于促进像素级别的 图像特征表达. 因此, Wang 等 ${ }^{[7]}$ 提出双阶段图像级 要素驱动网络, 其采用图像级别预训练和像素级 微调的经典训练方式. 该方法提供了弱监督下训练 显著性检测器的新范式, 并且使用现有的大规模 图像级标签数据集对网络进行预训练; 其次，提出 全局平滑池化层深人优化前景推理网络(foreground inference networks, FIN), 充分利用图像级标签的 信息预测显著性图.

直接从图像级标签精准地提取语义特征具有 一定的难度，因此目前弱监督方法都很难进一步 挖掘图像中的潜在信息，如图像的高级语义信息、 物体存在依赖关系等特征. 基于上述问题，本文提 出一种利用语义的单词映射作为自训网络的显著 性弱标签、逐级诱导提取显著性特征的方法. Xu 等 ${ }^{[8]}$ 利用注意力机制将图像中的单词映射可视化. 受其启发, 本文通过较强的残差网络提取图像的 基本特征后, 通过长短期记忆 (long short-term memory, LSTM)网络进一步提炼图像特征, 并根据 已知的文本语义标签进行监督驱动，从中获得具 有图像描述能力的显著性弱标签数据集. 当然, 仅 仅通过单词语义驱动并不能得到较为真实的显著 性图. 为此采用由简单数据集到复杂数据集逐渐 过渡的方式诱导网络，这种训练策略的设计方式 使得网络的自适应能力逐渐加强. 主流的弱监督 标签更新方式是将上一阶段产生的特征作为下一 阶段特征训练的监督标签，但是没有很好地评判
指标指导，此方式很容易使网络向坏的方向训练. 因此，采用余弦相似度阈值对监督标签进行标记 和更新，根据不同的相似度阈值有针对性地进行 标签更新. 与其他弱监督显著性检测方法相比，本 文方法具有更强的显著性特征提取能力.

本文主要创新点如下:

(1) 使用鱼网络进行特征提取, 并在网络的高 级语义端添加注意力模型作为特征聚合引导机制, 同时使用余弦相似度阈值对语义标签进行更新迭 代，从而获得更好的特征表示.

(2) 网络训练采用由简单数据集到复杂数据 集逐渐诱导的训练策略, 使得网络逐步具备强大 的特征提取能力.

(3) 基于图像描述网络 ${ }^{[8]}$ 对图像的语义预测描 述, 构建单词语义映射热力图, 获得显著性弱监督 标签数据集.

\section{1 相关工作}

弱监督学习吸引了越来越多研究人员的关注, 如目标检测、语义分割及边界检测等，相关的数据 呈现爆炸式的增长. 但是, 对于网络训练, 有效的 数据并不是很多, 标注带来的极大成本让人们开 始探索新的训练方式，用廉价的类别数据诱导不 同任务的网络训练，以极大地节约标签成本.

由于图像级标签太稀疏，无法产生精确的像 素级显著性特征，因此传统的无监督显著性检测 方法利用轮廓信息自动生成嘈杂的显著性特征图, 逐步完善并用于像素级监督训练，从而得到更加 有效的深层显著性检测模型. Zhou 等 ${ }^{[9]}$ 提出一个通 用的技术，称为类激活映射 (class activation mapping, CAM). 这使得经过分类训练的卷积神经网 络(convolutional neural networks, CNN)能够在不使 用任何边界的情况下完成各种任务. CAM 能够对 图像中显著的部分进行可视化, 在任何给定的图 像上, 突出显示被 CNN 检测的识别对象部分. $\mathrm{Li}$ 等 ${ }^{[10]}$ 从深层 $\mathrm{CNN}$ 中提取多尺度特征来表示超像 素, 并使用分类器网络预测每个超像素的显著性 得分. Hou 等 ${ }^{[11]}$ 提出一种具有深度监督的跳级结构 用于显著性检测. Ahn 等 ${ }^{[12]}$ 利用 CAM 来训练网络 预测局部图像区域内的语义亲和力，然后将其合 并, 以更新 CAM 并生成分割标签. Wang 等 ${ }^{[7]}$ 提出 一种弱监督学习方法, 仅使用图像级标签进行显 著性检测, 通过 FIN 与全卷积网络(fully convolu- 
tional networks, FCN)联合训练图像级标签预测, 使 FCN 可以将对象类别标签分配给相应的对象区 域，而 FIN 可以预测所有潜在前景区域的显著性 图; 最后利用条件随机场(conditional random field, CRF)加强空间标签的一致性，进一步提高网络对 显著性物体的检测性能. $\mathrm{Li}$ 等 ${ }^{[13]}$ 通过使用类别标 签训练一个 FCN 后, 利用启发式的无监督显著性 检测方法获得噪声特征矩阵, 并将粗粘的特征图 和得分最高的 3 个 CAM 进行整合，通过 CRF 技术 获得较好的轮廓边界; 之后将 $\mathrm{CRF}$ 处理后的特征 矩阵热力图作为训练标签进行网络的后续自训, 并逐步得到理想的结果. Kolesnikov 等 ${ }^{[14]}$ 提出一种 加权排序层来聚合空间响应，有针对性地分割训 练类别的对象. Zeng 等 ${ }^{[15]}$ 提出一个统一的框架来 训练具有多种弱监督来源的显着性检测模型. Qiu 等 ${ }^{[16]}$ 提出一种新颖的深度 CRF 网络，同时考虑深 度特征和邻居信息多尺度特征. Chen 等 ${ }^{[17]}$ 利用视 觉注意力机制和残差细化，提出一种有效的全卷积 显著目标检测网络. Chi 等 ${ }^{[18]}$ 通过将高级和低级特 征与联合概率估计相结合，利用聚类和结构化随机 森林等方法进一步细化了显著性图的局部细节.

图像理解的目的是生成语法上合理的语句来 描述图像内容. 现有的图像字幕模型 ${ }^{[19-21]}$ 都受益 于 CNN 和 LSTM, 其中 CNN 用于编码图像内容的 信息，而 LSTM 用于将其转换为语句. $\mathrm{Xu}$ 等 $^{[8]}$ 利用 LSTM 隐藏状态来表达空间注意力，并进一步用于 下一个单词的预测. Sun 等 ${ }^{[22]}$ 为像素级或区域级预 测任务设计出一个参数较少、性能较优的骨干结 构. Oktay 等 ${ }^{[23]}$ 提出一种能自动聚焦不同形状和大 小物体的注意力机制模型. Qin 等 ${ }^{[24]}$ 提出一个预测优化的框架，以及一个针对边界感知显著目标检 测的混合损失函数, 该框架由一个编码器解码器 网络和一个残差优化模块构成，分别负责显著预 测和特征图优化，并使用金字塔的注意力机制和 显著边缘来进行显著性检测. Wang 等 ${ }^{[25]}$ 使用金字 塔的注意力机制和显著边缘模块分等级地细化显 著性特征图.

当前主流的弱监督训练方式大多数都是通过 CAM，逐渐地诱导自训网络并逐步加强特征的提 取方式. 不同于上述的方法, 本文方法更加注重理 解图像，通过文本的弱驱动获得显著性边缘轮廓， 利用廉价的分类标签数据集训练基础性的多任务 网络参数, 以有效地节约标签手工标注成本. 为了 更好地利用基础性参数，提出基于知识的逐渐促
进机制的训练策略, 利用简单数据集和复杂数据 集的双重促进, 使得网络的特征表达能力越来越 强. 这种思路在网络的初期训练是很有效的, 即诱 导式的训练效果更明显.

\section{2 本文方法}

本文方法主要包括 2 个步骤:

Step1. 如图 1 所示, 将训练数据集输人到图像描述 网络, 生成语义映射图作为初始标签.

Step2. 采用鱼网络 ${ }^{[22]}$ 作为主干网络, 并在末端加 人注意力机制, 采用余弦相似度阈值进行目标标签的更 新迭代, 逐渐训练网络.



图 1 弱标签单词语义映射网络结构图

\section{1 图像语义特征映射}

图像除了色彩纹理能带来视觉上的刺激之外, 不同的图像所表达的语义能够使读者产出幻想, 如美丽的黄昏、清澈的日出以及隐隐约约的山间小 道. 在没有图像之前, 可以联想颜色和纹理, 因此 在图像显著性特征的研究中, 高级语义是非常重 要的文本特征. $\mathrm{Xu}$ 等 ${ }^{[8]}$ 利用注意力机制实现图像 描述的功能，并在实验过程中对生成单词的注意 力进行可视化映射, 得到了图像中的一些高级语 义特征. 受其启发, 将需要生成显著性标签的图片 输人到如图 1 所示一个已经训练好的带有注意力 机制的图像描述网络中, 输人的图片首先经过一 个残差网络(编码器部分)生成 $n$ 维特征图; 然后将 其输人到一个带有注意力机制的 LSTM(解码器部 分)中, 最后生成一句话(即输人图像的描述). 在生 成描述中每一个单词时，通过注意力机制获得对 应于特征图的权重向量, 在特征矩阵和权重向量 的相互映射下，将得到每个单词的特征注意力映 射. 如图 2 所示, 小猫咪的轮廓在不同的单词描述 中所呈现的特征表示也不同, 单词 $\mathrm{a}$ 所体现的仅仅 是小猫咪最模糊的位置信息，类似于物体的定位; 而单词 close 描述的是猫的形状是小的. 映射图中 所表现的特征基本上符合猫的小型的轮廓, 但并 不是很清晰. 然而在经过不同的语义上下文联系 后, 当描述 cat 时, 表达的物体轮廓更加地接近物 体真实形状, 这些映射图是比较理想的弱监督标 签, 从中选出第 2 个单词的映射图(因为第 2 个 


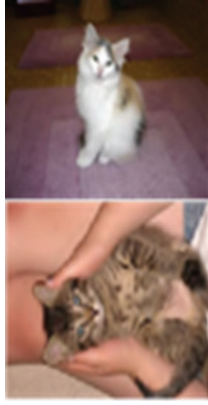

a. 原图

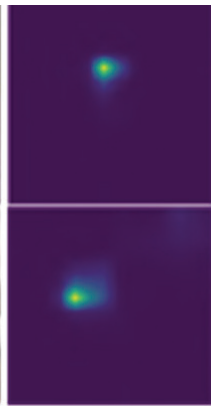

b. a/a

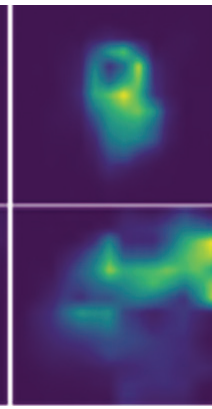

c. cat/close

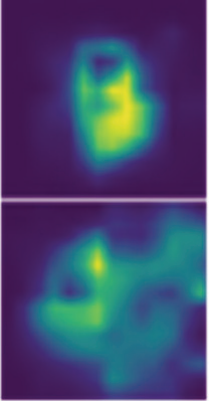

d. sit/person

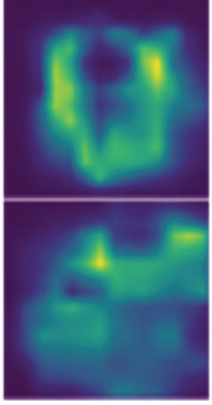

e. on/hold

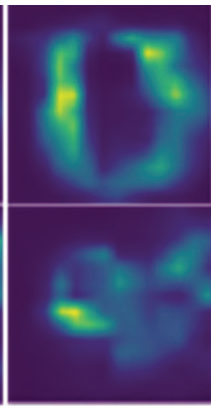

f. $a / a$

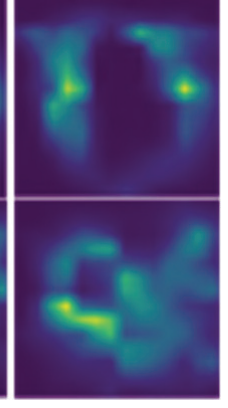

g. rug/cat

图 2 单词语义热力图映射效果图

单词一般为图像中显著性物体类别的描述)作为后 续特征自训的初始标签, 通过图像语义特征映射 阶段将得到具有语义的显著性弱标签数据集，该 数据集将用作第 2.2 节自训网络.

\section{2 特征自训网络}

本文主要采用鱼网络作为特征自训网络的主 干网络, 通过迁移学习对已经在分类数据集中训 练好的参数进行自适应的参数微调, 使其更加适 合不同的任务. 仅仅依靠主干网络不能很好地进 行特征表达, 需要对其提取过程进行有效的特征 引导. 如图 3 所示, 仅使用深度单流结构整个网络 结构呈现鱼形，因此主要由鱼头、鱼尾及鱼身体 3 部分构成. 其网络训练数据可以表示为 $D=$ $\left\{\left(X_{t}, Y_{t}\right), t=1,2, \cdots, T\right\}$. 其中, $X_{t}$ 表示原始的图像 输人; $Y_{t}$ 表示每幅原始图像所对应的二值化标签. 为了更加简洁地表示, 用下角标 $t$ 表示了原始输人
图像的个数. 下面将详细地描述网络结构的细节, 并通过图和公式的形式表达相互关系, 网络最终 输出 $y$, 可以表示为 $y=A\left(y^{\prime}\right)$. 其中, $y^{\prime}=F(X, \gamma)$ 表示鱼网络的特征输出; $X$ 表示鱼网络的特征输人; $\gamma$ 表示鱼网络训练参数; $A$ 表示注意力机制. 为了 节约设计成本, 引用文献[23]提出的注意力机制, 将鱼网络末端的不同尺度的图像作为注意力模型 的特征输人, 如图 3 所示 $x_{1}$ 和 $x_{2}$ 分别是鱼网络的 倒数第 3 层和倒数第 4 层的输出. 在经过小型感受 野降维后将其特征合并, 并将得到的特征经过激 活函数处理后与原始的输入信息进行概率相乘运 算，使得不同尺度的信息之间具有一定的相互引 导性，从而得到具有共同联系的显著性特征表示. 最后将注意力机制得到的预测特征图 $y$ 与目标标 签 $Y$ 进行交叉熵运算, 并进行损失函数优化, 得到 $L=-Y \ln y-(1-Y) \ln (1-y)$.



图 3 特征自训网络结构图

\section{3 弱标签更新算法}

弱标签更新算法步骤如下:

输人. 初始数据集 $Y$.

Step1. 判断预测特征图 $y$ 与目标标签 $Y$ 的余弦相 似度阈值.
Step2. 根据阈值选择对监督标签更新(Yes)或不更 新(No).

Step3. 更新后将保留的标签或更新的标签作为下次 迭代的新监督标签 $Y$, 重复 Step1 Step2 直至训练收玫.

Step4. 当网络训练达到收玫时, 更换复杂数据集 
进行反复训练, 重复 Step1 Step3 直至再次收玫.

Step5. 当更换数据集后并收玫时, 网络训练结束.

弱标签更新算法将已知的单词语义特征图作 为最初的监督标签, 通过设置的余弦相似度阈值

$$
\cos \theta=\frac{\sum_{i=1}^{n}\left(y_{i} \times Y_{i}\right)}{\sqrt{\sum_{i=1}^{n}\left(y_{i}\right)^{2}} \times \sqrt{\sum_{i=1}^{n}\left(Y_{i}\right)^{2}}}
$$

对特征矩阵进行标签驱动更新. 其中， $y_{i}$ 表示当 前阶段网络的特征预测图中的像素值; $Y_{i}$ 表示上 一阶段的网络的特征预测图中的像素值; $n$ 表示 预测特征图中像素点的个数; $\cos \theta$ 表示 2 个预测 图的相似程度, 该值越接近于 1 , 表示 2 个特征图 越相似. 不更新大于 $\cos \theta$ 阈值的预测特征, 反之 进行更新; 通过不断重复此过程, 使得网络越来越 趋向于表达显著性物体的清晰轮廓, 如图 4 所示.
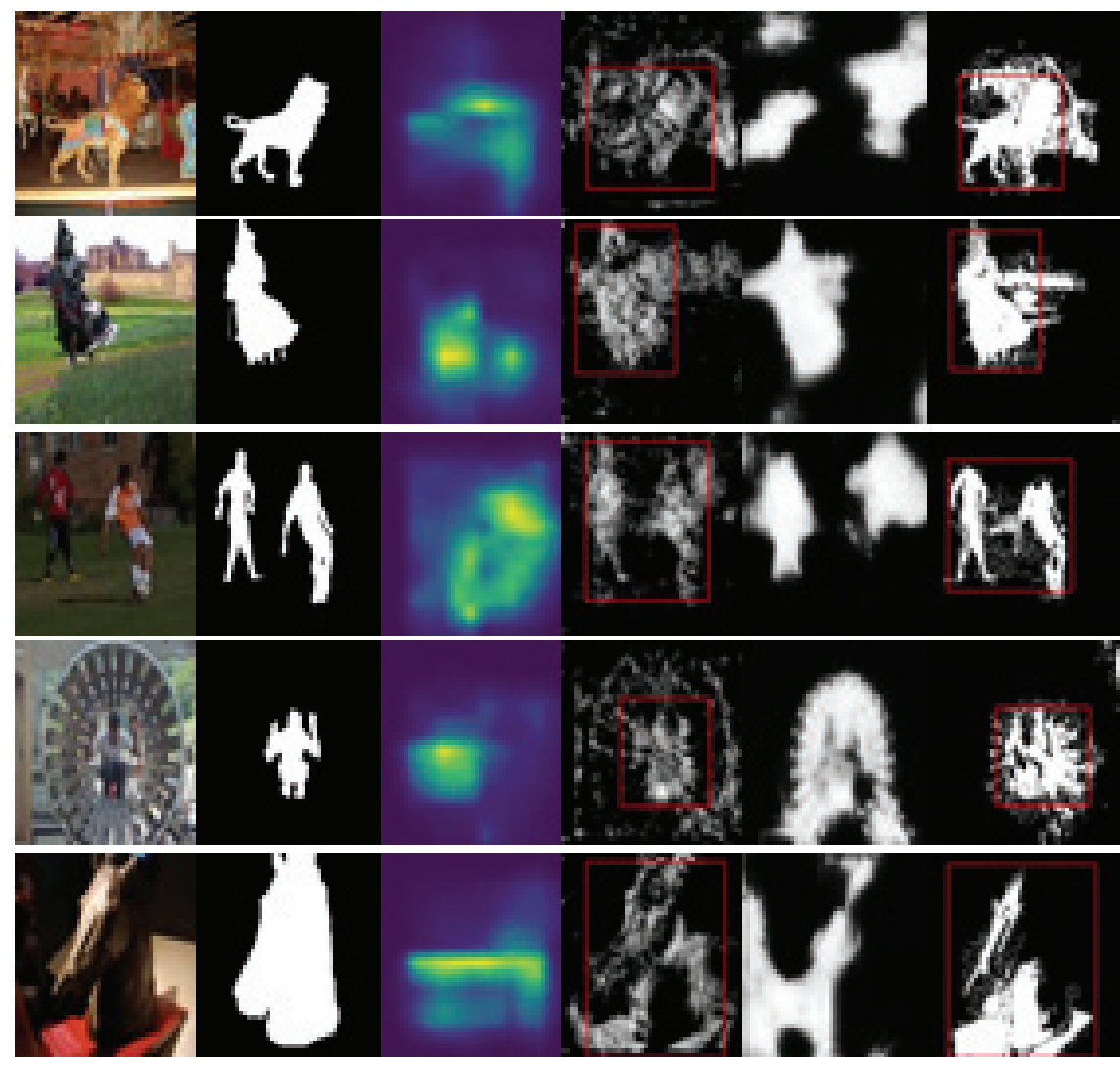

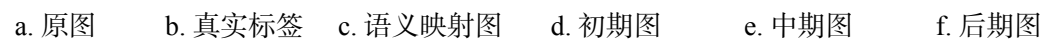

图 4 语义映射显著性检测效果图

如图 4d 所示, 此时显著性物体的基本轮廓已 经确定，但是由于最初的监督标签并不是很好，因 此, 此时的物体的显著性图比较模糊. 但是随着训 练数据集变得复杂, 经过网络的诱导性训练后, 显 著性图逐渐清晰. 从效果图中可以看出, 随着训练 的深人，图像中显著性图逐渐得到更清晰、更准确 的结果.

\section{3 实 验}

\section{1 数据集}

网络自训阶段使用由简单到复杂的数据集逐 渐训练网络, 将物体轮廓和背景很明显的图像集
划分成简单数据集, 将背景比较复杂并且物体轮 廓不容易识别的图像划分到复杂数据集. 其中, 简 单数据集包括数据集 ECSSD 和从互联网上搜集的 1000 张自然图片, 其中每张图片物体的前景和背 景很明显, 通过视觉观察很容易识别和区分. 采用 数据集 DUTS 作为训练过程中的复杂数据集, DUTS 是个大型的显著性检测数据集，包括 10553 张训练图片和 5019 张测试图片. 测试数据集包括 DUTS-TEST, HKU-IS, DUT-OMRONO 和 PASCAL-S 数据集, DUT-OMRONO 包括 5168 张相对复杂背 景的图片, PASCAL-S 包括 850 张来自 PASCAL VOC 2010 数据集中的图片, HKU-IS 包括 4447 张 复杂场景图片. 


\section{2 实验细节及评价指标}

整个实验是通过 Pytorch 框架实现的，实验环 境是 NVIDIA GTX1080Ti GPU 显卡, 弱监督标签 构成中使用 $\mathrm{COCO}$ 语义数据集. 采用原数据集中 标准的训练集和测试集, 采用 Adam 优化器, 训练 次数大概 100 次, 每批样本个数为 32 , 微调学习率 为 0.00001 . 在自训网络中, 采用 ECSSD, DUTS 和 简单数据集进行训练, 测试使用 DUTS-TEST, HKU-IS, DUT-OMRONO 以及 PASCAL-S 数据集, 优化器采用 Adam, 其中学习率为 0.000001 , 批 样本个数为 $2, \mathrm{CRF}$ 的微调参数为 50 和 5 , 余弦 相似度的阈值设置范围为 $0.6 \sim 0.8$, 迭代次数 50 70 次.

\section{3 结果分析}

在实验过程中，与部分显著性方法进行了对 比实验, 其中包括 2 种无监督方法 $\mathrm{DRFI}^{[26]}$ 和

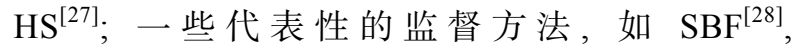
$\mathrm{MCDL}^{[29]}, \mathrm{LEGS}^{[30]}, \mathrm{MDF}^{[31]}$ 和弱监督方法 $\mathrm{WSS}^{[7]}$.
不同显著性方法的效果图如图 5 所示，可以看出， 与其他方法相比, 基于语义的弱监督显著性检测 的定位精度和显著性边界(如第 3 行飞机的显著性 图)均优于其他方法. 表 1 所示为在基准数据集上 的 $F$ 值和 MAE 评估. 从表 1 可以看出, 该方法在 各个基准数据集整体上表现比较出色. 通过实验 数据发现, 本文方法整体上优于监督方法 SBF 和 弱监督方法 WSS. 仅仅利用图像中的文本语义信 息作为弱监督标签有一定的难度，与其他的方法 相比, 本实验思路是新颖的, 取得的结果依赖于网 络模型的 2 个创新点, 一个是利用图像中的语义产 生的热力映射图作为训练标签, 另一个是在训练 网络的过程中采用由简单到复杂的数据集逐渐过 渡的训练诱导策略. 在对比实验的指标中, 主要 是利用了 $F$-MAX 值和 MAE 值进行评价, 本文方 法在 4 个数据集上 $F$-MAX 值均表现良好, 而 MAE 稍差一些. 通过在不同数据集上的实验数据能够很 明显地看出，本文方法整体上取得了更好的结果.

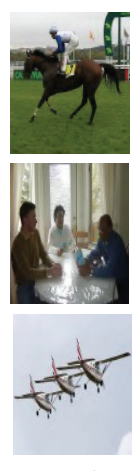

原图


真实标签

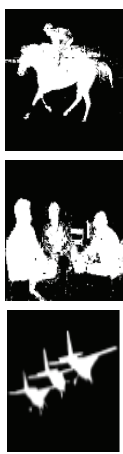

本文方法
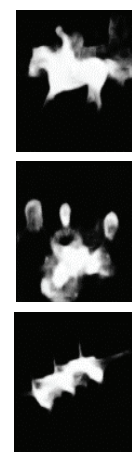

$\mathrm{SBF}^{[28]}$
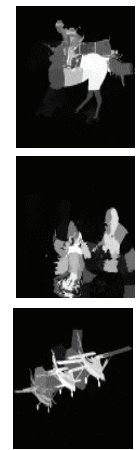

$\mathrm{MCDL}^{[29]}$


LEGS $^{[30]}$
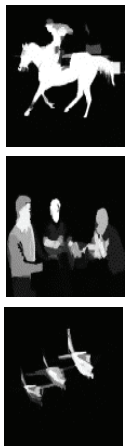

$\mathrm{MDF}^{[31]}$

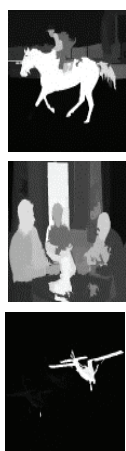

$\mathrm{HS}^{[27]}$
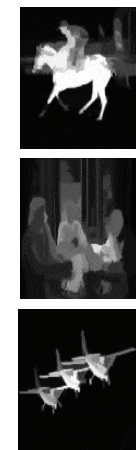

$\operatorname{DRFI}^{[26]}$

图 5 弱监督各方法结果对比

表 1 不同方法在 4 个数据集上的性能比较

\begin{tabular}{|c|c|c|c|c|c|c|c|c|}
\hline \multirow{2}{*}{ 方法 } & \multicolumn{2}{|c|}{ DUTS-TEST } & \multicolumn{2}{|c|}{ HKU-IS } & \multicolumn{2}{|c|}{ DUT-OMRONO } & \multicolumn{2}{|c|}{ PASCAL-S } \\
\hline & $F$-MAX & MAE & $F$-MAX & MAE & $F$-MAX & MAE & $F$-MAX & MAE \\
\hline 本文 & 0.679 & 0.115 & 0.821 & 0.071 & 0.659 & 0.111 & 0.728 & 0.137 \\
\hline $\mathrm{SBF}^{[28]}$ & 0.657 & 0.109 & 0.821 & 0.078 & 0.649 & 0.110 & 0.726 & 0.133 \\
\hline $\mathrm{MCDL}^{[29]}$ & 0.634 & 0.105 & 0.787 & 0.092 & 0.670 & 0.089 & 0.706 & 0.143 \\
\hline LEGS $^{[30]}$ & 0.612 & 0.137 & 0.736 & 0.119 & 0.631 & 0.133 & & \\
\hline $\operatorname{MDF}^{[31]}$ & 0.657 & 0.114 & 0.839 & 0.129 & 0.643 & 0.092 & 0.704 & 0.142 \\
\hline $\mathrm{HS}^{[27]}$ & 0.504 & 0.243 & 0.652 & 0.215 & 0.561 & 0.227 & 0.569 & 0.262 \\
\hline $\mathrm{DRFI}^{[26]}$ & 0.600 & 0.155 & 0.745 & 0.145 & 0.623 & 0.150 & 0.639 & 0.207 \\
\hline $\mathrm{WSS}^{[7]}$ & 0.657 & 0.100 & 0.822 & 0.079 & 0.602 & 0.110 & 0.724 & 0.141 \\
\hline
\end{tabular}

注. 粗体表示最优结果.

\section{4 结 语}

图像的颜色、纹理和方向等信息是基本的低级 信息，而高级别语义关系是图像所包含的高级信
息. 本文提出了基于单词语义的映射图作为弱监 督标签来进行网络自训的弱监督显著性方法. 不 同的映射图表达不同的语义信息, 通过选择描述 物体语义较全面的热力图作为弱标签, 在自训网 
络中采用余弦相似度阈值进行目标标签的更新迭 代，并在主干网络鱼网络末端添加注意力机制，可 有效地对特征进行引导. 本文方法对图像中显著 性物体的整体部分有较好的检测效果，为以后的 弱监督显著性物体检测提供了一个思路. 但是对 于边缘复杂的物体，检测出的边缘轮廓精度不足. 如何在弱监督下实现边缘复杂物体的精细检测问 题将是未来的工作.

\section{参考文献(References):}

[1] Huang Z L, Wang X G, Wang J S, et al. Weakly-supervised semantic segmentation network with deep seeded region growing[C] //Proceedings of the IEEE Conference on Computer Vision and Pattern Recognition. Los Alamitos: IEEE Computer Society Press, 2018: 7014-7023

[2] Xiao H X, Feng J S, Lin G S, et al. Monet: deep motion exploitation for video object segmentation[C] //Proceedings of the IEEE Conference on Computer Vision and Pattern Recognition. Los Alamitos: IEEE Computer Society Press, 2018: 1140-1148

[3] Zhao R, Ouyang W L, Wang X G. Unsupervised salience learning for person re-identification[C] //Proceedings of the IEEE Conference on Computer Vision and Pattern Recognition. Los Alamitos: IEEE Computer Society Press, 2013: 3586-3593

[4] Zhang J M, Sclaroff S, Lin Z, et al. Minimum barrier salient object detection at $80 \mathrm{fps}[\mathrm{C}] / /$ Proceedings of the IEEE International Conference on Computer Vision. Los Alamitos: IEEE Computer Society Press, 2015: 1404-1412

[5] Kong Y Q, Wang L J, Liu X P, et al. Pattern mining saliency[C]//Proceedings of European Conference on Computer Vision. Heidelberg: Springer, 2016: 583-598

[6] Long J L, Zhang N, Darrell T. Do convnets learn correspondence?[C] //Proceedings of the Advances in Neural Information Processing Systems. Cambridge: MIT Press, 2014: 1601-1609

[7] Wang L J, Lu H C, Wang Y F, et al. Learning to detect salient objects with image-level supervision[C] //Proceedings of the IEEE Conference on Computer Vision and Pattern Recognition. Los Alamitos: IEEE Computer Society Press, 2017: 136-145

[8] Xu K, Ba J, Kiros R, et al. Show, attend and tell: Neural image caption generation with visual attention[C] //Proceedings of International Conference on Machine Learning. New York: JMLR Press, 2015: 2048-2057

[9] Zhou B L, Khosla A, Lapedriza A, et al. Learning deep features for discriminative localization[C] //Proceedings of the IEEE Conference on Computer Vision and Pattern Recognition. Los Alamitos: IEEE Computer Society Press, 2016: 2921-2929

[10] Li G B, Yu Y Z. Visual saliency based on multiscale deep features[C] //Proceedings of the IEEE Conference on Computer Vision and Pattern Recognition. Los Alamitos: IEEE Computer Society Press, 2015: 5455-5463

[11] Hou Q B, Cheng M M, Hu X W, et al. Deeply supervised salient object detection with short connections[C] //Proceedings of the IEEE Conference on Computer Vision and Pattern Recognition. Los Alamitos: IEEE Computer Society Press, 2017:
3203-3212

[12] Ahn J, Kwak S. Learning pixel-level semantic affinity with image-level supervision for weakly supervised semantic segmentation[C] //Proceedings of the IEEE Conference on Computer Vision and Pattern Recognition. Los Alamitos: IEEE Computer Society Press, 2018: 4981-4990

[13] Li G B, Xie Y, Lin L. Weakly supervised salient object detection using image labels[OL]. [2020-04-15]. https://arxiv.org/ abs $/ 1803.06503$

[14] Kolesnikov A, Lampert C H. Seed, expand and constrain: Three principles for weakly-supervised image segmentation $[\mathrm{C}] / /$ Proceedings of European Conference on Computer Vision. Heidelberg: Springer, 2016: 695-711

[15] Zeng Y, Zhuge Y Z, Lu H C, et al. Multi-source weak supervision for saliency detection[C] //Proceedings of the IEEE Conference on Computer Vision and Pattern Recognition. Los Alamitos: IEEE Computer Society Press, 2019: 6074-6083

[16] Qiu W L, Gao X B, Han B. Saliency detection using a deep conditional random field network[J]. Pattern Recognition, 2020, 103: 107266

[17] Chen S H, Wang B, Tan X L, et al. Embedding attention and residual network for accurate salient object detection[J]. IEEE Transactions on Cybernetics, 2020, 50(5): 2050-2062

[18] Chi J N, Wu C D, Yu X S, et al. Saliency detection via integrating deep learning architecture and low-level features[J]. Neurocomputing, 2019, 352: 75-92

[19] Lu J S, Xiong C M, Parikh D, et al. Knowing when to look: Adaptive attention via a visual sentinel for image captioning $[\mathrm{C}] / /$ Proceedings of the IEEE Conference on Computer Vision and Pattern Recognition. Los Alamitos: IEEE Computer Society Press, 2017: 375-383

[20] Vinyals O, Toshev A, Bengio S, et al. Show and tell: a neural image caption generator[C] //Proceedings of the IEEE Conference on Computer Vision and Pattern Recognition. Los Alamitos: IEEE Computer Society Press, 2015: 3156-3164

[21] Gu J X, Cai J F, Wang G, et al. Stack-captioning: coarse-to-fine learning for image captioning[OL]. [2020-04-15]. https://arxiv. org/abs/1709.03376

[22] Sun S Y, Pang J M, Shi J P, et al. Fishnet: a versatile backbone for image, region, and pixel level prediction[C] //Proceedings of the Advances in Neural Information Processing Systems. Cambridge: MIT Press, 2018: 754-764

[23] Oktay O, Schlemper J, Folgoc L L, et al. Attention U-net: Learning where to look for the pancreas[OL]. [2020-04-15]. https://arxiv.org/abs/1804.03999

[24] Qin X B, Zhang Z C, Huang C Y, et al. BASNet: boundary-aware salient object detection[C] //Proceedings of the IEEE Conference on Computer Vision and Pattern Recognition. Los Alamitos: IEEE Computer Society Press, 2019: 7479-7489

[25] Wang W G, Zhao S Y, Shen J B, et al. Salient object detection with pyramid attention and salient edges[C]//Proceedings of the IEEE Conference on Computer Vision and Pattern Recognition. Los Alamitos: IEEE Computer Society Press, 2019: 1448-1457

[26] Jiang H Z, Wang J D, Yuan Z J, et al. Salient object detection: a discriminative regional feature integration approach[C] // Proceedings of the IEEE Conference on Computer Vision and Pat- 
tern Recognition. Los Alamitos: IEEE Computer Society Press, 2013: 2083-2090

[27] Yan Q, Xu L, Shi J P, et al. Hierarchical saliency detection[C] // Proceedings of the IEEE Conference on Computer Vision and Pattern Recognition. Los Alamitos: IEEE Computer Society Press, 2013: 1155-1162

[28] Peng H W, Li B, Ling H B, et al. Salient object detection via structured matrix decomposition[J]. IEEE Transactions on Pattern Analysis and Machine Intelligence, 2017, 39(4): 818-832

[29] Zhao R, Ouyang W L, Li H S, et al. Saliency detection by multi-context deep learning[C]//Proceedings of the IEEE Con- ference on Computer Vision and Pattern Recognition. Los Alamitos: IEEE Computer Society Press, 2015: 1265-1274

[30] Wang L J, Lu H C, Ruan X, et al. Deep networks for saliency detection via local estimation and global search[C] // Proceedings of the IEEE Conference on Computer Vision and Pattern Recognition. Los Alamitos: IEEE Computer Society Press, 2015: 3183-3192

[31] Li G B, Yu Y Z. Visual saliency based on multiscale deep features[C] //Proceedings of the IEEE Conference on Computer Vision and Pattern Recognition. Los Alamitos: IEEE Computer Society Press, 2015: 5455-5463 